\title{
Selective Maintenance for Multi-state \\ Series-parallel Systems under Economic Dependence
}

\author{
Cuong D. Dao, Ming J. Zuo and Mayank Pandey \\ Department of Mechanical Engineering, University of Alberta, \\ Edmonton, Alberta, T6G 2G8, Canada
}

\begin{abstract}
This paper presents a study on selective maintenance for multi-state series-parallel systems with economically dependent components. In the selective maintenance problem, the maintenance manager has to decide which components should receive maintenance activities within a finite break between missions. All the system reliabilities in the next operating mission, the available budget and the maintenance time for each component from its current state to a higher state are taken into account in the optimization models. In addition, the components in series-parallel systems are considered to be economically dependent. Time and cost savings will be achieved when several components are simultaneously repaired in a selective maintenance strategy. As the number of repaired components increases, the saved time and cost will also increase due to the share of setting up between components and another additional reduction amount resulting from the repair of multiple identical components. Different optimization models are derived to find the best maintenance strategy for multi-state series-parallel systems. A genetic algorithm is used to solve the optimization models. The decision makers may select different components to be repaired to different working states based on the maintenance objective, resource availabilities and how dependent the repair time and cost of each component are.
\end{abstract}


Key words: Selective maintenance, multi-state, series-parallel system, reliability, maintenance time, multiple repairs, economic dependence.

\section{Introduction}

Many systems in the industry are required to perform several missions with finite breaks between missions. In order to maintain good conditions of the systems, we have to decide which subsystems or components should be given maintenance activities in the limited time allotted between missions. This problem is called selective maintenance.In this study, the selective maintenance problem for multi-state series-parallel systems under strict requirements on resources such as time and cost is considered. The first selective maintenance model was introduced in 1998 by Rice et al. [1]. In their paper, the mathematical model for optimizing the reliability of binary-state series-parallel systems with independently and identically distributed (i.i.d.) time to failure of the components in each subsystem and a heuristic rule was provided to solve the model. Then, different selective maintenance models and applications for binary state case were developed by Cassady et al., 2001, [2]. Schneider and Cassady, 2004, [3] extended the model by Rice et al. by considering selective maintenance for a fleet including multiple binary series-parallel systems. Pandey et al., 2013, [4] studied the selective maintenance for binary systems considering imperfect repair, in which the health of components may be not "as good as new" and depends on the maintenance cost. Maillart et al., 2009, [5] investigated selective maintenance optimization for binary series-parallel systems working under multiple identical missions and found that the difference in the long-run performance between optimal single-mission policy and multi-mission policy is minimal.

In all these studies, [1] - [5], the system and its components are assumed to be in two possible states of failed or functioning. However, in practice, many systems and 
components can operate in more than two possible states, e.g. "partial failed" or "imperfect functioning". In this more general case, Chen et al., 1999, [6] modeled selective maintenance for multi-state systems with the objective of minimizing the cost of maintenance subject to reliability constraints. Liu and Huang, 2010, [7] presented a selective maintenance model for systems with multi-state corresponding to cumulative performance of $N$ binary components and considering the imperfect maintenance that may restore the condition of the system to an intermediate state.

In the study of Chen et al. [6], a selective maintenance model for the multi-state series-parallel system was provided. In this model, the maintenance cost is minimized subjected to the system reliability constraints. However, in maintenance management and particularly in selective maintenance, the time allocated for maintenance activities is one of the most critical constraints. This paper studies the model by Chen et al. [6] for a situation in which the maintenance crews have to consider the available time for total maintenance activities, $T_{0}$, which is limited.

Furthermore, many maintenance actions are usually implemented on multi-component systems in a selective maintenance scenario. In all previous selective maintenance studies, [1]-[7], components in the system are assumed to be economically independent, i.e. the repair of each component is independent to other components. However, in many industrial systems such as aircrafts, medical equipments, automotive mechanical machines and nuclear power plants, etc., repairing multiple components, especially identical components, is always more economical due to the share of setting up, tools, materials and labor. In this case, those components in such systems are considered to be economically dependent. The idea of setting up cost saving due to performing a group of maintenance activities that may require only one set-up was employed in a series of papers [8] - [12] and a review by Dekker et al., 1997, [13]. More recently, Nourelfath and Chatelet, 2012, [14] investigated economic 
dependence and structural dependence between components of a parallel system in the production and preventive maintenance planning problem with the objective of minimizing the total production and maintenance cost. Maaroufi et al. [15] considered maintenance for binary systems with propagated failures and economic dependence where a fixed "set-up cost" for dismantling and reassembling the system is incurred only one time when more than one components is replaced. In these papers, they considered the set-up cost incurred each time there is corrective, preventive or opportunistic replacement and the components are assumed to be "as good as new" after replacement, rather than the possibility of repairing components to different intermediate states as in multi-state systems. Moreover, these papers did not consider time savings when performing maintenance on multiple components in the systems.

This paper, therefore, focuses on modeling two types of economic dependency between multi-state components based on the share of setting up and the advantage of repairing multiple identical components in each subsystem of the multi-state series-parallel system. Both time and cost savings can be assured when several components are selected to be repaired in a selective maintenance strategy. In this study, the system and its components may be in any state from a set of all possible states, $S=\{0,1, \ldots, K\}$, where state $K$ is perfect functioning, state 0 is complete failure, others are intermediate states. The multi-state series-parallel systems are assumed to have the following characteristics:

- All the components in a subsystem are identical and s-independent.

- The maintenance activities do not make the condition of components and the system worse, i.e. the states of the system and its components are not lower after going out of the maintenance depot.

- The resource requirement for a single maintenance activity is deterministic and known. 


\section{Problem formulation}

\section{Notation:}

$M: \quad$ Number of subsystems connected in series

$i: \quad$ Subsystem index, $i \in\{1,2, \ldots, M\}$

$N_{i}: \quad$ Number of components in subsystem $i$

$j: \quad$ Component index in each subsystem, $j \in\left\{1,2, \ldots, N_{i}\right\}$

$(i, j)$ : Component $j$ of subsystem $i, i \in\{1,2, \ldots, M\}, j \in\left\{1,2, \ldots, N_{i}\right\}$

$K$ : $\quad$ Maximum state of each component as well as the system

$a, b, k:$ State indexes of component or system, $0 \leq a, b, k \leq K$

$Y_{i j}: \quad$ Given state of component $(i, j)$ at the end of the previous mission $0 \leq Y_{i j} \leq K$

$Y: \quad$ State vector of all components in the system at the end of the previous mission

$X_{i j}: \quad$ State of component $(i, j)$ at the end of maintenance period $Y_{i j} \leq X_{i j} \leq K$

$X: \quad$ State vector of all components in the system at the end of maintenance period

$\phi_{s}: \quad$ The state function of the system after the next operating mission, $\phi_{s} \in\{0,1, \ldots, K\}$

$\tau: \quad$ The operating mission duration

$t_{i j}(a, b)$ : The required time for individual repair (single repair) of component $(i, j)$ from state $a$ to state $b$; if all components in a subsystem are i.i.d.: $t_{i j}(a, b)=t_{i}(a, b)$, and: $t_{i j}(a, b)=\left\{\begin{aligned} 0 & \text { if } b \leq a \\ >0 & \text { otherwise }\end{aligned}\right.$

$T_{i}$ : The time matrix for repairing a single component in subsystem $i$ from state $a$ to state $b$

$t_{i}^{\prime}(a, b)$ : The adjusted repair time of repairing an additional identical component in subsystem $i$ from state $a$ to state $b$

$f_{T}^{i}(a, b)$ : The time saving coefficient when repairing multiple components in subsystem $i$ from state $a$ to state $b$ 
$\Delta t_{s}:$ The amount of time saved per component due to the share of setting up

$T_{C}: \quad$ The total completion time of all selective maintenance activities

$T_{0}: \quad$ The available time for all selective maintenance activities

$c_{i j}(a, b)$ :The cost for individual repair of component $(i, j)$ from state $a$ to state $b$; if all components in a subsystem are i.i.d.: $c_{i j}(a, b)=c_{i}(a, b)$, and:

$$
c_{i j}(a, b)=\left\{\begin{aligned}
0 & \text { if } b \leq a \\
>0 & \text { otherwise }
\end{aligned}\right.
$$

$c_{i}^{\prime}(a, b)$ : The adjusted repair cost for each i.i.d. component in subsystem $i$ from state $a$ to state $b$

$C_{i}$ : The cost matrix for single repair of a component in subsystem $i$ from state $a$ to state $b$

$\Delta c_{s}:$ The amount of saved money per component due to the share of setting up

$f_{C}^{i}(a, b)$ :The cost saving coefficient when repairing multiple components in subsystem $i$ from state $a$ to state $b$

$C_{m}^{i}: \quad$ The total adjusted cost of repairing $m$ identical components in subsystem $i$ from state $a$ to state $b$

$C_{T}: \quad$ The total cost of all selective maintenance activities

$C_{0}: \quad$ The available budget for all selective maintenance activities

$\beta$ : The exponents indicating the rate of time/cost improvement of identical repairs characterized by type of component in a subsystem

$p_{i j}(b, a)$ : The probability of a component $(i, j)$ being in state $b$ at the beginning of an operating mission and being in state $a$ at the end of the mission. If all components in a subsystem are i.i.d. $p_{i j}(b, a)=p_{i}(b, a)$, and:

$$
p_{i j}(b, a)=\left\{\begin{aligned}
0 & \text { if } b<a \\
>0 & \text { otherwise }
\end{aligned}\right.
$$

$P_{i}$ : The transition matrix of a component in subsystem $i$ 
$R_{s}(k)$ : The probability that the system state is greater than or equal to $k, P\left(\phi_{s} \geq k\right)$ or the reliability of the system at level $k$

$R_{0}: \quad$ The minimum reliability requirement of the system

\subsection{Multi-state series-parallel system and selective maintenance problem description}

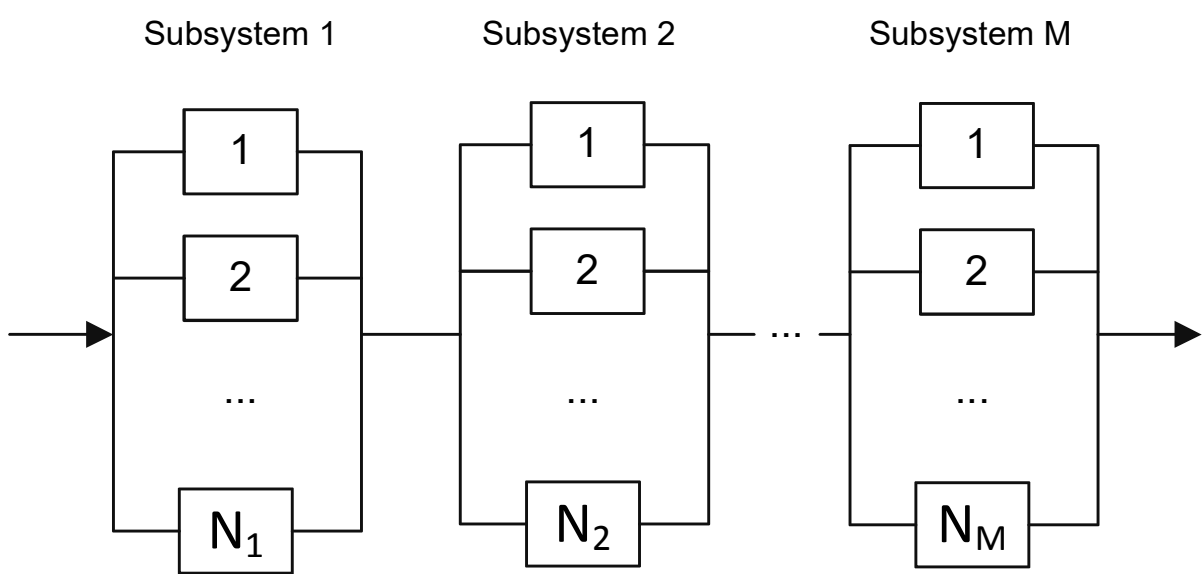

Figure 1. Series-Parallel System

The series-parallel system consists of $M$ independent subsystems connected in series and in subsystem $i, i=\{1,2, \ldots, M\}$, there are $N_{i}$ identical components connected in parallel (Figure 1). Each component and the system may be one in a set of $K+1$ possible states, $S=\{0,1, \ldots, K\}$, where state $K$ is perfect functioning, state 0 is complete failure, others are intermediate states. In a series-parallel structure, the state of a subsystem is the maximum state among its components and the system state is determined by the minimum state among $M$ subsystems. In the selective maintenance problem, the system has to work consecutively identical missions with break interval of $T_{0}$ - time from the end of previous mission to the beginning of the next mission. The maintenance crews have to decide what and how to maintain each component in the system within available budget $C_{0}$ and available time $T_{0}$.

For each component $(i, j), Y_{i j}, 0 \leq Y_{i j} \leq M$, represents its state at the time of entering the maintenance depot and $X_{i j}$ is its state after the selective maintenance break. Because the 
maintenance activities do not make the condition of components and the system worse, it is clear that $Y_{i j} \leq X_{i j} \leq M$. In the selective maintenance problem, the state vector of all components in the system at the time of entering the maintenance depot, $Y=\left\{Y_{i j}\right\},\left(i=1,2, \ldots, M, j=1,2, \ldots, N_{i}\right)$, is known; we have to find its state vector at the time of exiting the maintenance depot, $X=\left\{X_{i j}\right\},\left(i=1,2, \ldots, M, j=1,2, \ldots, N_{i}\right)$.

\subsection{The system reliability}

A component degrades with use, and its state at the end of an operating mission is a random variable. Xue and Yang, 1995, [16] investigated the measure of evaluating components' performance degradation in multi-state coherent systems. This may serve as a good reference for the state transition analysis of multi-state components and systems. Here, we will not focus on the component's degradation process in the operating mission time $\tau$. This paper assumes that the probabilities for a component in subsystem $i$ at any pre-specified state $b$ degrading to all possible state $a(0 \leq a \leq b)$ after the operating mission, $p_{i}(b, a)$, are already known. When $b=0,1, \ldots, K$, these probabilities form a $(K+1) \times(K+1)$ transition probability matrix of each component in subsystem $i$ for completing a mission as given in equation (1).

$$
P_{i}=\left[\begin{array}{cccc}
1 & 0 & \ldots & 0 \\
p_{i}(1,0) & p_{i}(1,1) & \ldots & 0 \\
\ldots & \ldots & \ldots & \ldots \\
p_{i}(K, 0) & p_{i}(K, 1) & \ldots & p_{i}(K, K)
\end{array}\right], i=1,2, \ldots, M
$$

In equation (1), the state of components cannot rise after an operating mission, i.e. $p_{i}(b, a)=0$ if $b<a$. If a component begins in state $0, a=0$, it will remain in that state after the next mission since it cannot degrade further, hence $p_{i}(0,0)=1$. If a component is in 
state $b, 0<b \leq K$, its state after the next mission can be any value from the set of $\{b, b-1, \ldots, 0\}$, therefore:

$$
\sum_{a=0}^{K} p_{i}(b, a)=\sum_{a=0}^{b} p_{i}(b, a)=1 \text { for } b=0,1, \ldots, K
$$

In order to deal with the selective maintenance problem, we need to find the system reliability at the end of the next mission at a specified level $a$, i.e. the required reliability level, $R_{s}(a)=P\left(\phi_{s} \geq a\right)$. In series-parallel structures, a subsystem is in a state less than $a$, $\phi_{\text {subi }}<a$, when all of its components are in states less than $a$. The event that the subsystem $i$ is in state $a$ or above at the end of the next mission, $\phi_{\text {subi }} \geq a$, is the complement event of $\phi_{\text {subi }}<a$. Therefore, the reliability of the system at level $a, R_{s}(a)$, can be computed by using equation (3), Chen et.al. [6].

$$
\begin{aligned}
R_{s}(a) & =P\left(\phi_{s} \geq a\right)=\prod_{i=1}^{M} P\left(\phi_{\text {subi }} \geq a\right)=\prod_{i=1}^{M}\left(1-P\left(\phi_{\text {subi }}<a\right)\right) \\
& =\prod_{i=1}^{M}\left(1-\prod_{j=1}^{N_{i}} \sum_{k=0}^{a-1} p_{i}\left(X_{i j}, k\right)\right)
\end{aligned}
$$

\subsection{The system repair time and cost}

When entering the maintenance depot, components may be in any state from the set of state space, i.e. each elements of $Y_{i j}$ can be any number from $\{0,1, \ldots, K\}$. Within the maintenance break duration, $T_{0}$, and the available budget, $C_{0}$, the system and its components are subjected to be maintained to "properly working" states so that the system will meet the reliability requirement in the next operating mission. Here, the required time and cost for single repair (individual repair) of an i.i.d. component in subsystem $i$ from state $a$ to any state $b$ which is greater than $a$ are known. The single repair time and cost of each component 
in subsystem $i$ from an arbitrary state $a$ to state $b$ are arranged in matrix form as in (4) and (5) respectively.

$$
\begin{aligned}
T_{i} & =\left[\begin{array}{cccc}
0 & t_{i}(0,1) & \ldots & t_{i}(0, K) \\
0 & 0 & \ldots & t_{i}(1, K) \\
\ldots & \ldots & \ldots & \ldots \\
0 & 0 & \ldots & t_{i}(K-1, K) \\
0 & 0 & \ldots & 0
\end{array}\right], i=1,2, \ldots, M \\
C_{i} & =\left[\begin{array}{cccc}
0 & c_{i}(0,1) & \ldots & c_{i}(0, K) \\
0 & 0 & \ldots & c_{i}(1, K) \\
\ldots & \ldots & \ldots & \ldots \\
0 & 0 & \ldots & c_{i}(K-1, K) \\
0 & 0 & \ldots & 0
\end{array}\right], i=1,2, \ldots, M
\end{aligned}
$$

In (4) and (5), the time and cost of repairing a single component from state $a$ to state $b$ are non-zero values when $a$ is less than $b$. In general, $t_{i}(a, b)<t_{i}\left(a, b^{\prime}\right)$ and $c_{i}(a, b)<c_{i}\left(a, b^{\prime}\right)$ if $b<b^{\prime} ; t_{i}(a, b)=0, c_{i}(a, b)=0$ if $a=b$. Without doubt to the calculation of maintenance time and cost, an element in these matrices is set equal to zero if $a$ is greater than $b$, i.e. $t_{i}(a, b)=0, c_{i}(a, b)=0$ if $a>b$.

In order to calculate the total repair time and cost of the entire system, it is necessary to analyze the relationship of the repair time and cost between components. The time and cost for improving the state of a component may not affect that of other components independent repair time for each component. However, the same setting up, maintenance process, equipments, etc. may be utilized when multiple components are simultaneously selected to be repaired in a maintenance strategy. In this case, time and cost savings will be achieved by repairing multiple components in the selective maintenance modeling.

\subsubsection{Independent repair time and cost}


When the repair time for each component is independent, the total time required to do the selective maintenance for the system is simply a summation of all the individual repair time of its components in (4). If we know the components' state vector at the time of entering the maintenance $\operatorname{depot} Y=\left\{Y_{i j}\right\},\left(i=1,2, \ldots, M, j=1,2, \ldots, N_{i}\right)$, the total system maintenance time corresponding to a vector of component state at the time of exiting the maintenance depot, $X=\left\{X_{i j}\right\}$, can be computed and represented as in equation (6).

$$
T_{C}\left(X_{i j}\right)=\sum_{i=1}^{M} \sum_{j=1}^{N_{i}} T_{i}\left(X_{i j}, Y_{i j}\right)
$$

Similarly, the total system maintenance cost can be obtained by taking the sum of all single components' repair costs.

$$
C_{T}\left(X_{i j}\right)=\sum_{i=1}^{M} \sum_{j=1}^{N_{i}} C_{i}\left(X_{i j}, Y_{i j}\right)
$$

\subsubsection{Dependent repair time and cost}

In most realistic systems, time and cost savings are achieved when multiple components are selected to be repaired in a maintenance strategy, especially for identical components in each subsystem of series-parallel systems. They usually require similar initial setting up, labor and equipments. Here, the concept of "set-up cost" [13] is employed to both cases of time and cost. In addition, it is even more economical when repairing multiple identical components in the same current state (condition) $a$ to the same properly working state $b$. This is because there are not only the share of setting up but also the advantages of ordering materials (batch order) and using the same process of performing maintenance on those identical components. Additional cost and time savings for this type of repair should be addressed. Therefore, we consider two types of time and cost savings of repairing multiple 
components in series-parallel systems as follows:

1. Time and cost savings due to the share of setting up.

2. Additional reduction time and cost of repairing multiple identical components in a subsystem from the same current state $a$ to the same properly working state $b$.

In the section below we will focus on formulating the total actual repair cost for a selective maintenance strategy based on the single repair cost matrix in (5) with the consideration of two types of cost saving above. Once the total repair cost is determined, the total maintenance time of the system can be calculated accordingly.

The savings due to the share of setting up are assumed to be fixed per component since it is associated with the process of preparation for maintenance such as erecting, dismantling and reassembling the system, etc. Thus, in the first type of dependency, a fixed amount of "set-up cost", $\Delta c_{s}$, is saved whenever an additional component is selected in a selective maintenance strategy (Figure 2). The more components to be maintained, the more money is saved due to the share of setting up. If $N_{r}$ components in the system are maintained in a selective maintenance strategy, the total amount of money saved due to the share of setting up will be $\left(N_{r}-1\right) \times \Delta c_{s}$. 


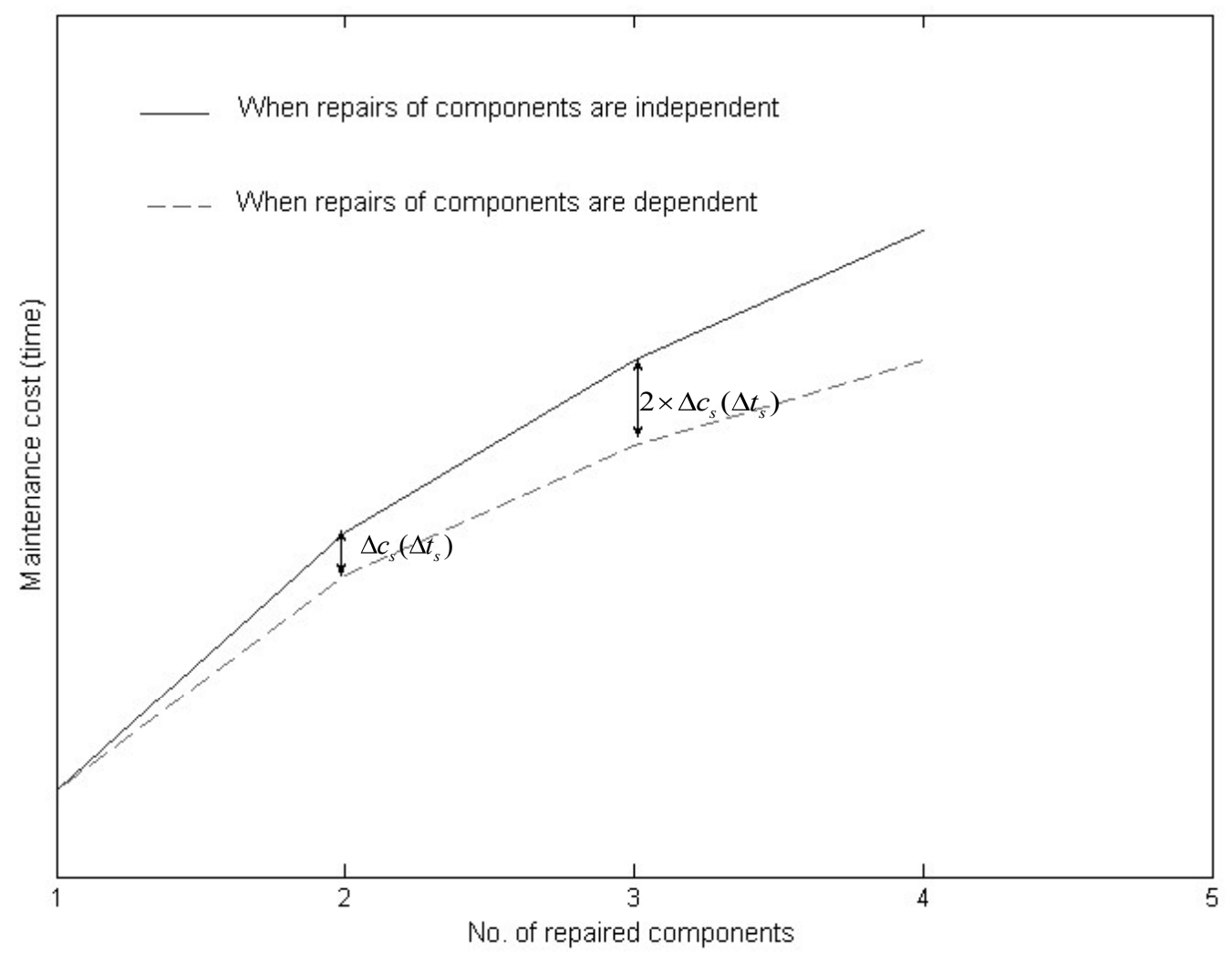

Figure 2.The maintenance cost (time) vs. number of repaired components when there is the share of setting up

Now, let's consider repairing $m$ identical multi-state components in a subsystem $i$ with $c_{i}(a, b)$ being the cost for individually repairing each component from state $a$ to state $b$. In multiple repairs of identical components, the same technology and equipment can be utilized, and the maintenance is more efficient in repetitive tasks. Thus, in addition to the cost saved by setting up sharing, we introduce a cost saving coefficient, $f_{C}^{i}(a, b)$, to represent the repairing cost dependency of multiple identical components in subsystem $i$. Denote $c_{i}^{\prime}(a, b)$ to be the adjusted repair cost for an additional component in subsystem $i$ from state $a$ to state $b$. The calculations of total maintenance cost and the saved amount due to each type of dependencies are illustrated in Figure 3. The adjusted repair cost and the saved amount in addition to a fixed set-up cost for repairing a component from state $a$ to state $b$ are calculated by (8) and (9) respectively. 


$$
\begin{aligned}
& c_{i}^{\prime}(a, b)=f_{C}^{i}(a, b) \times c_{i}(a, b)-\Delta c_{s} \\
& c_{i}(a, b)-c_{i}^{\prime}(a, b)-\Delta c_{s}=\left(1-f_{C}^{i}(a, b)\right) \times c_{i}(a, b)
\end{aligned}
$$

From (8), we can form an adjusted repair cost matrix for a component in each subsystem $i$, $C_{i}^{\prime}$; each element in this matrix is a function of $f_{C}^{i}$ and $\Delta c_{s}$. The total adjusted cost for repairing $m$ identical components from state $a$ to state $b$ can be computed as in (10).

$$
\begin{aligned}
C_{m}^{i} & =c_{i}(a, b)+(m-1) \times c_{i}^{\prime}(a, b) \\
& =c_{i}(a, b)+(m-1) \times f_{C}^{i}(a, b) \times c_{i}(a, b)-(m-1) \times \Delta c_{s}
\end{aligned}
$$

Theoretically, $f_{C}^{i}$ can take any value between 0 and 1 . When the two components are independent, $f_{C}^{i}=1$, the total repair cost is simply a summation of components' repair cost. $f_{C}^{i}=0$ when we do not need any additional cost to repair an extra component other than the first one. 


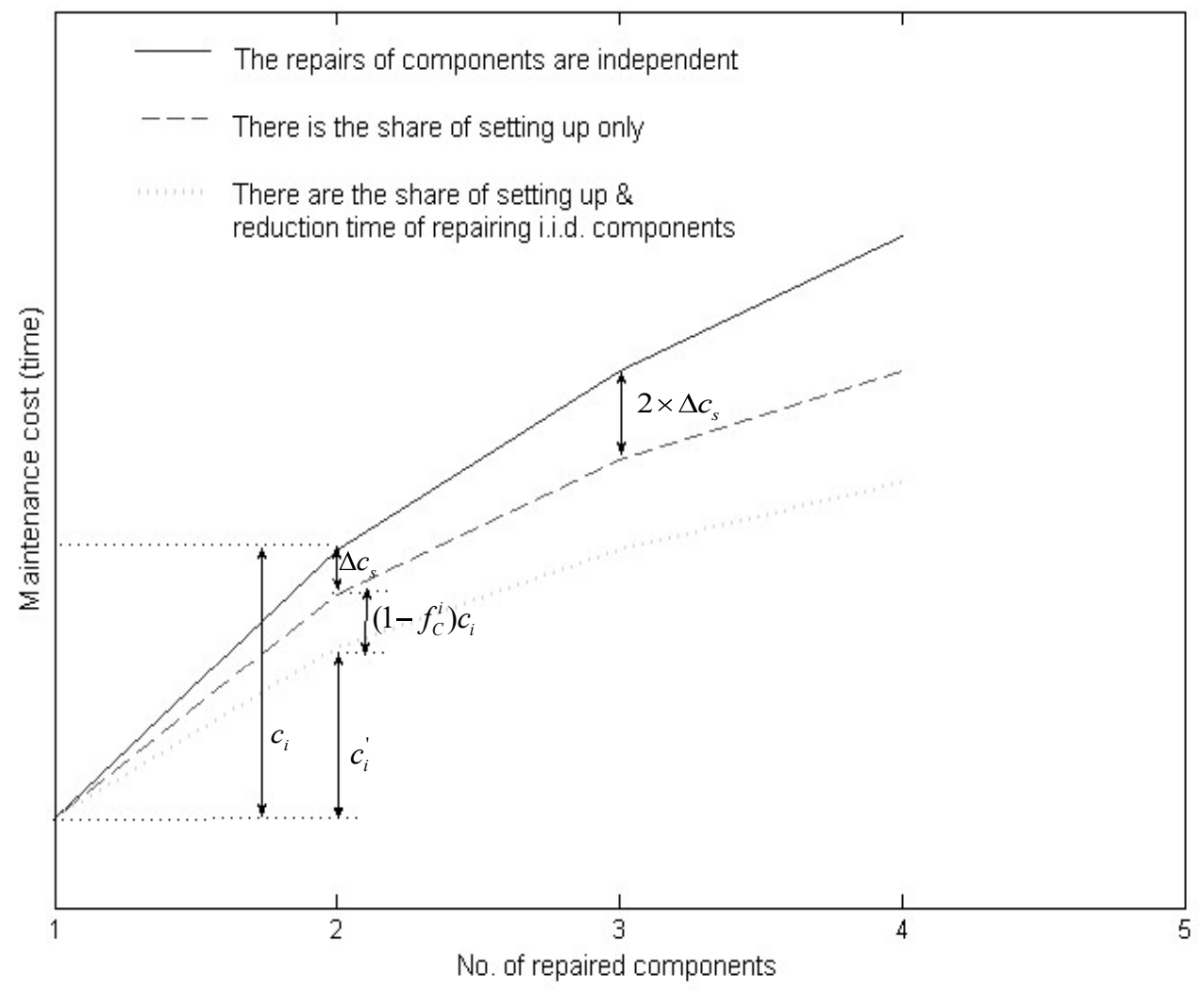

Figure 3. The maintenance cost when repairing identical components

The total system maintenance cost is, then, the summation of total adjusted repair cost of each component. It is a function of the decision variables $X_{i j}$, the amount of saved money due to the share of setting up - $\Delta c_{s}$, the cost saving coefficients - $f_{C}^{i}$ and the components' state at the time of entering and exiting the maintenance depot.

$$
C_{T}\left(X_{i j}\right)=\sum_{i=1}^{M} \sum_{j=1}^{N_{i}} C_{i j}^{\prime}\left(X_{i j}, Y_{i j}, \Delta c_{s}, f_{C}^{i}\right)
$$

In (11), $C_{i j}^{\prime}\left(X_{i j}, Y_{i j}, \Delta c_{s}, f_{C}^{i}\right)$ is the adjusted repair cost of component $(i, j)$ which is equivalent to $c_{i}^{\prime}(a, b)$ in the explanation above. In the selective maintenance problem for the series-parallel system considering economic dependence, we need an assumption that the components in the same subsystem have to be maintained in a group to gain the benefits of 
time and cost savings. However, in the process of calculation, it is no doubt to assume a subsystem is repaired before another sub-system since all the results on the system reliabilities, cost and time are not affected. With this assumption, if there are multiple components in different sub-systems subjected to be maintained in a selective maintenance strategy, the value of the adjusted repairing for the first component is always equal to the cost of the single repair for that component. For the next component, $c_{i}^{\prime}(a, b)$ is calculated as in (12).

$$
c_{i}^{\prime}(a, b)=\left\{\begin{array}{cc}
f_{C}^{i} \times c_{i}(a, b)-\Delta c_{s} & \text { If there is identical repair } \\
c_{i}(a, b)-\Delta c_{s} & \text { Otherwise }
\end{array}\right.
$$

Similarly, the total system maintenance time for a selective maintenance strategy can be obtained if the time saved due to the share of setting up - $\Delta t_{s}$, the time dependent coefficients - $f_{C}^{i}$, and the state of component at the time of entering the maintenance depot are known.

$$
T_{C}\left(X_{i j}\right)=\sum_{i=1}^{M} \sum_{j=1}^{N_{i}} T_{i j}^{\prime}\left(X_{i j}, Y_{i j}, \Delta t_{s}, f_{T}^{i}\right)
$$

where $T_{i j}^{\prime}$ is the adjusted repair time for component $j$ in subsystem $i$ when the repairs of components are dependent.

In order to understand more about the proposed saving model, we also investigate other saving mechanisms and their effects on the selective maintenance for the multi-state series-parallel system. The saving patterns of performing multiple activities when task repetitions take place will be investigated and compared to our saving model. The basic saving model is known as "power model" or "Log-linear model" [17]. It has the following mathematical representation: 


$$
C(x)=C_{1} \times x^{-\beta} \text { or } \log C(x)=\log C_{1}-\beta \log x,
$$

where $C_{1}$ is the cost (time) of the first unit, $C(x)$ is the average cost (time) of $x$ units, and $\beta$ is an exponent indicating the rate of productivity improvement, $0 \leq \beta \leq 1 . \beta=0$ means that there is no advantage of performing multiple tasks and when $\beta$ is close to 1 , the benefit of multiple tasks execution is larger.

From the basic model in equation (14), several models have been developed to measure the average unit cost/time as a function of the number of units produced. These models are summarized in [18]. In this paper, we will consider three typical saving patterns: the Log-linear, Plateau and Exponential models.

$$
\begin{aligned}
& C(x)=C_{p}+C_{1} x^{-\beta} \\
& C(x)=C_{1} x^{-\beta} e^{c x}
\end{aligned}
$$

The Plateau model in (15) is often applied when there is a limit of the unit cost as $x$ increases to a very big value. $C_{p}$ is a parameter representing the plateau limit of the average unit cost. In (16), $c$ is another parameter, usually a constant that reflects the upturn effect on the cost when the number of units increases to a considerably large value.

From the average unit cost, we can find the total cost of repairing $m$ identical components, $C_{m}^{i}$, in a sub-system of the series-parallel system. Table 1 summarizes $C_{m}^{i}$ calculated from the proposed model and three saving models in the literature. 
Table 1. Summary of different cost saving models of repairing $m$ identical components

\begin{tabular}{|c|c|c|}
\hline Model & Average cost of $\mathbf{x}$ units & $\begin{array}{c}\text { Total cost of repairing } \boldsymbol{m} \text { identical } \\
\text { components }\end{array}$ \\
\hline Log-linear & $C_{1} x^{-\beta}$ & $C_{m}^{i}=C_{1} m^{-\beta+1}$ \\
\hline Plateau & $C+C_{1} x^{-\beta}$ & $C_{m}^{i}=C m+C_{1} m^{-\beta+1}$ \\
\hline Exponential & $C_{1} x^{-\beta} e^{c x}$ & $C_{m}^{i}=C_{1} m^{-\beta+1} e^{c m}$ \\
\hline Proposed model & {$\left[C_{1}+(x-1) C_{1}^{\prime}\right] / x$} & $C_{m}^{i}=C_{1}+(m-1) C_{1}^{\prime}$ \\
\hline
\end{tabular}

In the calculation of average cost of $x$ units for the proposed model in Table 1, we use $C_{1}^{\prime}$ as the adjusted repair cost, which can be computed in the same way as in (12). We use three saving patterns in Table 1 for describing the dependent relationship between the cost/time of multiple repairs and the number of components involved in each sub-system. The results of selective maintenance for multi-state series-parallel systems and discussions will be provided in Section 4.

\subsection{Mathematical programming formulation}

Cassady et al, 2001, [2], proposed mathematical programming models for optimizing selective maintenance of binary systems. This paper extends those models to the multi-state case. The selective maintenance problem for multi-state series parallel system is a non-linear integer programming problem with known components' characteristics such as the state of components at the time of entering the maintenance depot, the time and cost of single repair, time and cost savings of multiple repair, state probability distribution of each component in the next mission.

In a very popular selective maintenance problem, the maintenance crews have to find what maintenance activities associated with each component to be performed to achieve the 
maintenance objective of increasing the system reliability under limitation of resources such as time and cost. This type of problem can be formulated as follows:

$$
\begin{aligned}
& \text { P1: } \begin{array}{l}
\text { Maximize } f= \\
R_{s}(a)=\prod_{i=1}^{M}\left(1-\prod_{j=1}^{N_{i}} \sum_{k=0}^{a-1} p_{i}\left(X_{i j}, k\right)\right) \\
\text { Subject to: } \quad T_{C}\left(X_{i j}\right) \leq T_{0} \\
C_{T}\left(X_{i j}\right) \leq C_{0} \\
Y_{i j} \leq X_{i j} \leq K \\
X_{i j} \text { is integer }, i=1,2, \ldots, M ; j=1,2, \ldots, N_{i}
\end{array}
\end{aligned}
$$

In the model, the objective function (17) is to maximize the reliability of the system at a specified working level $a$, which has been formulated in section 2.2. There are two types of constraints in (18) and (19) which restrict the total time and cost for all maintenance activities within available time, $T_{0}$, and budget, $C_{0}$. The component repair time and cost may be or may be not independent. We can find the total time and cost for maintaining the system to desired working level as explained in section 2.3. The decision variables, $X_{i j}$, are the states of components at the time of exiting the maintenance depot. Since the maintenance activities do not worsen the state of the components, $X_{i j}$ must be integer value between $Y_{i j}$ and the maximum state $K$ for all $i=1,2, \ldots, M ; j=1,2, \ldots, N_{i}$.

The decision makers can use the problem P1 when they have information about their available time and budget and the system reliability is very critical. The solution of $\mathbf{P} \mathbf{1}$ gives us the most reliable system with on-hand resources. In practice, we may face many other situations of the principal objective and resource availability. When the total completion time is the most important issue it is treated as the objective of the selective maintenance problem. Then, the system reliability and cost are two constraints, and we have an 
alternative P2. Similarly, another derivation, P3, can be obtained as follows:

P2: $\quad$ Minimize $f=T_{C}\left(X_{i j}\right)$

Subject to: $\quad R_{s}\left(X_{i j}\right) \geq R_{0}$

$$
C_{T}\left(X_{i j}\right) \leq C_{0}
$$

$$
Y_{i j} \leq X_{i j} \leq K
$$

$$
X_{i j} \text { is integer, } i=1,2, \ldots, M ; j=1,2, \ldots, N_{i}
$$

P3: $\quad$ Minimize $f=C_{T}\left(X_{i j}\right)$

Subject to: $\quad R_{s}\left(X_{i j}\right) \geq R_{0}$

$$
\begin{aligned}
& T_{C}\left(X_{i j}\right) \leq T_{0} \\
& Y_{i j} \leq X_{i j} \leq K \\
& X_{i j} \text { is integer, } i=1,2, \ldots, M ; j=1,2, \ldots, N_{i}
\end{aligned}
$$

In the problems $\mathbf{P 2}$ and $\mathbf{P 3}$, the constraint (23) requires the reliability of the system to be greater than or equal to a specified level $R_{0}$. Both $R_{s}\left(X_{i j}\right)$ and $R_{0}$ are vectors with $K$-dimension; each dimension is the probability that the multi-state system is at the corresponding state or above.

For an extremely urgent situation, we have to maintain the system to attain a certain reliability level as soon as possible regardless of how costly it is. In this case, the constraints on cost will be released and a special case of problem $\mathbf{P 2}$ can be derived:

P4: $\quad$ Minimize $f=T_{C}\left(X_{i j}\right)$

Subject to: $\quad R_{s}\left(X_{i j}\right) \geq R_{0}$

$$
Y_{i j} \leq X_{i j} \leq K
$$




$$
X_{i j} \text { is integer }, i=1,2, \ldots, M ; j=1,2, \ldots, N_{i}
$$

It is also noted that many other derivations of these models can be obtained. Depending on the principal purpose of the maintenance and available information, the maintenance decision makers can select the most appropriate models and thereby understand what actions should be done based on his on-hand conditions.

\section{Solution approach}

Genetic Algorithms (GA) is used to solve the proposed selective maintenance models in section 2. In this section, we will provide a detailed solution representation for the selective maintenance problem, a general procedure of GA, and parameters setting in GA program. Further details on GA searching evolution and genetic operator's mechanisms can be found in [19] and [20].

\subsection{Solution representation}

Solution representation is one of the most important parts in the implementation of genetic algorithm. Let $N$ be the total number of components in the system, $N=N_{1}+N_{2}+\ldots+N_{M}$. The detail of solution representation is illustrated in Figure 4. Each chromosome consists of $N$ genes; each gene is an integer number between 0 and $K$ which represents the state of the corresponding component at the end of the maintenance break.

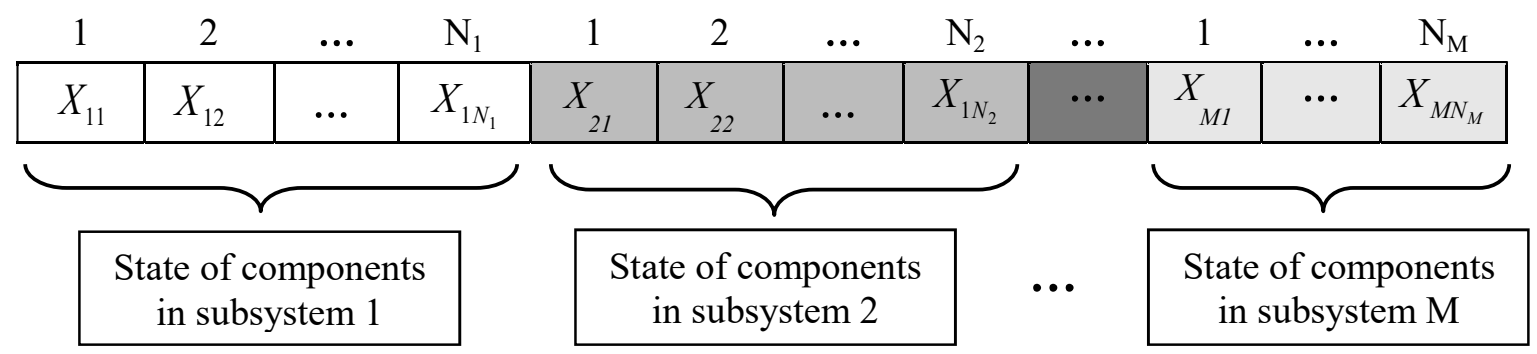

Figure 4. Solution representation 
All elements in the vector of components' state are ordered from subsystem 1 to subsystem $M$ and the decision variable, $X_{i j}, i=1,2, \ldots, N_{i}, j=1,2, \ldots, M$, can be transformed to the state vector $X_{i}, i=1,2, \ldots, N$. Each state vector is considered as a solution of the proposed selective maintenance problem. If the states of components at the time of entering the maintenance depot are known and can be rewritten in vector form, $Y_{i}, i=1,2, \ldots, N$,we can use GA to find the best combination of the components' outcome states after the maintenance break for the proposed optimal selective maintenance problem.

\subsection{General procedure and implementation of $G A$}

GA starts by randomly initializing a population of $N$ chromosomes in the first generation. Each chromosome represents a possible solution to the selective maintenance problem. Since each gene of chromosome represents the state of the corresponding component, we use a random generator to create each integer number in each gene within the range from $Y_{i}$ to $M$. The fitness function, i.e. the objective function, is used to evaluate the chromosomes, which allows a particular chromosome to be ranked against all the others. After computing the fitness values of the individuals, existing solutions are recombined using crossover, mutation, and reproduction procedures, to obtain new ones. The genetic algorithm terminates when a pre-specified number of generations is attained or a given time limit is over.

Based on this approach, the problem is coded using Matlab R2011a. The parameters setting for GA program is shown in Table 2. 
Table 2.GA parameters setting

\begin{tabular}{|c|c|c|}
\hline GA Parameters & $\begin{array}{c}\text { Value/ } \\
\text { Probability }\end{array}$ & Type \\
\hline Population size & 50 & - \\
\hline Crossover & 0.8 & Single point \\
\hline Mutation & 0.1 & Uniform \\
\hline Selection & - & Tournament \\
\hline $\begin{array}{c}\text { Number of GA iterations } \\
\text { (Stopping criteria) }\end{array}$ & 100 & - \\
\hline
\end{tabular}

\section{Illustrative examples, results and discussion}

Example 1: Considering a multi-state series-parallel (Figure 5) with $K=3, M=3, N_{l}=3, N_{2}$

$=2, N_{3}=4$, the given states of components when entering the maintenance depot are:

$$
Y_{i j}=\left[\begin{array}{llll}
2 & 0 & 1 & \\
2 & 2 & & \\
0 & 2 & 2 & 1
\end{array}\right] \text { or } Y=\left[\begin{array}{lllllllll}
2 & 0 & 1 & 2 & 2 & 0 & 2 & 2 & 1
\end{array}\right]
$$

For each i.i.d. component in subsystem $i, i=1,2,3$, the transition probability matrices and corresponding cost and time matrices for each individual maintenance activity are:

$$
\begin{gathered}
P_{1}=\left[\begin{array}{cccc}
1 & 0 & 0 & 0 \\
0.2 & 0.8 & 0 & 0 \\
0.1 & 0.3 & 0.6 & 0 \\
0.05 & 0.1 & 0.1 & 0.75
\end{array}\right], P_{2}=\left[\begin{array}{cccc}
1 & 0 & 0 & 0 \\
0.1 & 0.9 & 0 & 0 \\
0.1 & 0.3 & 0.6 & 0 \\
0.05 & 0.05 & 0.1 & 0.8
\end{array}\right], P_{3}=\left[\begin{array}{cccc}
1 & 0 & 0 & 0 \\
0.25 & 0.75 & 0 & 0 \\
0.05 & 0.15 & 0.8 & 0 \\
0.05 & 0.1 & 0.15 & 0.7
\end{array}\right] \\
C_{1}=\left[\begin{array}{llll}
0 & 7 & 8 & 10 \\
0 & 0 & 2 & 7 \\
0 & 0 & 0 & 2 \\
0 & 0 & 0 & 0
\end{array}\right], C_{2}=\left[\begin{array}{llll}
0 & 3 & 9 & 10 \\
0 & 0 & 6 & 8 \\
0 & 0 & 0 & 5 \\
0 & 0 & 0 & 0
\end{array}\right], C_{3}=\left[\begin{array}{lllc}
0 & 5 & 6 & 10 \\
0 & 0 & 4 & 8 \\
0 & 0 & 0 & 7 \\
0 & 0 & 0 & 0
\end{array}\right]
\end{gathered}
$$




$$
T_{1}=\left[\begin{array}{llll}
0 & 2 & 5 & 7 \\
0 & 0 & 3 & 4 \\
0 & 0 & 0 & 2 \\
0 & 0 & 0 & 0
\end{array}\right], T_{2}=\left[\begin{array}{llll}
0 & 3 & 4 & 6 \\
0 & 0 & 3 & 5 \\
0 & 0 & 0 & 2 \\
0 & 0 & 0 & 0
\end{array}\right], T_{3}=\left[\begin{array}{llll}
0 & 1 & 3 & 6 \\
0 & 0 & 2 & 4 \\
0 & 0 & 0 & 1 \\
0 & 0 & 0 & 0
\end{array}\right]
$$

The repairing time and cost for each component are independent. The required level of the system at the end of the next operating mission is $a=3$. We want to find the maximum reliability of the system in the next mission with available time $T_{0}=25$ time units, and total allowed budget $C_{0}=45$ cost units.
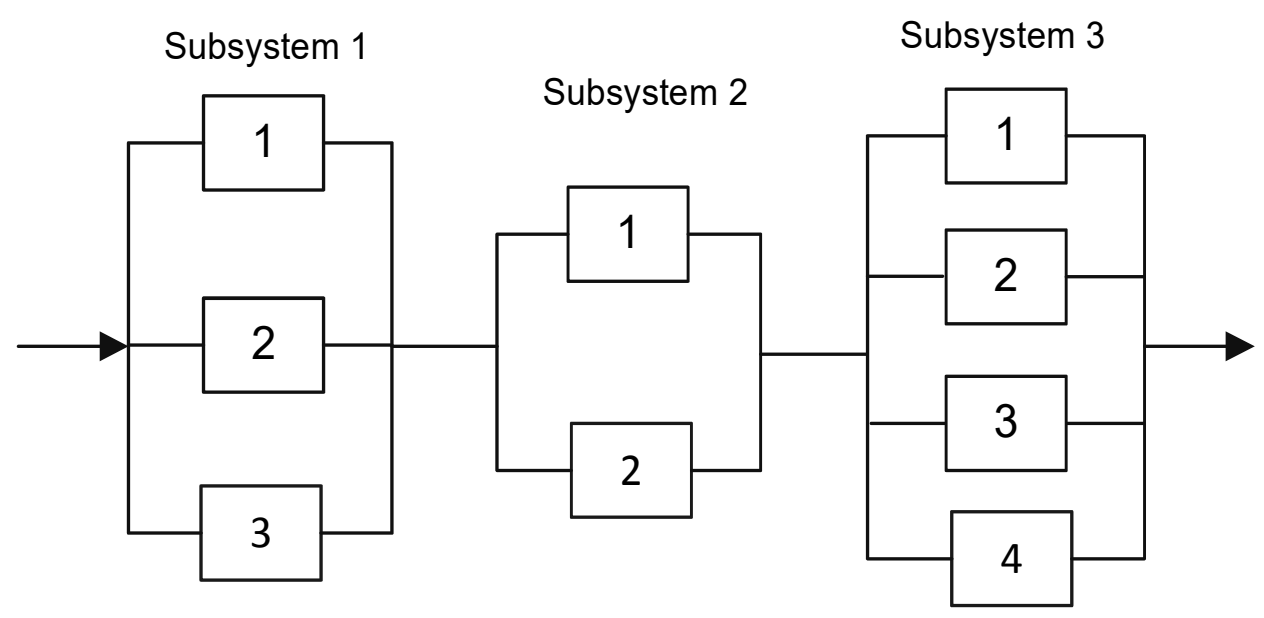

Figure 5. Series-parallel system example

By running the GA program to solve problem P1 to find the optimal reliability of the system at state $a=3$, we obtain $X=\left[\begin{array}{lllllllll}3 & 3 & 3 & 3 & 3 & 0 & 2 & 3 & 3\end{array}\right]$, that is, we select to repair all components in subsystem 1 and 2 to state 3 , do nothing to the first two components in subsystem 3 and repair the last two components in subsystem 3 to state 3 . 
Table 3. The optimal maintenance actions for all components

when repairs of components are independent (in problem P1)

\begin{tabular}{|c|c|c|c|c|}
\hline \multirow{2}{*}{ Subsystem $\boldsymbol{i}$} & \multicolumn{4}{|c|}{ Component $\boldsymbol{j}$} \\
\cline { 2 - 5 } & $\mathbf{1}$ & $\mathbf{2}$ & $\mathbf{3}$ & $\mathbf{4}$ \\
\hline $\mathbf{1}$ & $2 \rightarrow 3$ & $0 \rightarrow 3$ & $1 \rightarrow 3$ & - \\
\hline $\mathbf{2}$ & $2 \rightarrow 3$ & $2 \rightarrow 3$ & - & - \\
\hline $\mathbf{3}$ & $0-0$ & $2-2$ & $2 \rightarrow 3$ & $1 \rightarrow 3$ \\
\hline
\end{tabular}

The total cost for all maintenance activities is 44 cost units $(\leq 45)$ and the total maintenance time is $22(\leq 25)$ time units. Recall that $R_{s}(k)$ is the probability of the system state being in state $k$ or above in the next operating mission, we obtained the system optimal reliability at level $3, R_{S}(3)=0.85995$. The probabilities that the system will be at least in state 1 and 2 in the next mission are 0.99725 and 0.98222 respectively.

Table 4. The probability of the system being in state $k$ or above in the next operating mission (in problem Pl)

\begin{tabular}{|c|c|c|c|}
\hline$k$ & 1 & 2 & 3 \\
\hline$R_{S}(k)=P\left(\Phi_{S} \geq k\right)$ & 0.99725 & 0.98222 & 0.85995 \\
\hline
\end{tabular}

In example 1, solving problem $\mathbf{P 1}$ helps us find the most reliable system within available resources. Now, we want to investigate what maintenance actions should be implemented when the total maintenance time is the most critical issue. This situation is usually encountered in practice when a tight deadline is required in production or the next mission needs to be started as soon as possible. Example 2 is devoted to the illustration of this situation. With this example, the decision makers can find the best maintenance actions when time is the most critical while the required system reliability level is still close to the achieved results in Table 4.

Example 2: Consider the system in Example 1. Find the selective maintenance strategy that 
we can finish as soon as possible without any requirement on budget, but the system reliability at each level must be greater than $R_{0}(1)=0.99, R_{0}(2)=0.96, R_{0}(3)=0.85$.

Here, the cost constraint is released and we deal with problem $\mathbf{P 4}$ to minimize the total completion time of all selective maintenance activities with the constraint of achieving the required reliability. The solution of GA program results the optimal completion time of $\mathbf{1 8}$ time units. The vector state of all components at the end of the maintenance break is: $X=\left[\begin{array}{lllllllll}3 & 1 & 3 & 3 & 3 & 0 & 3 & 3 & 3\end{array}\right]$

Table 5. The optimal maintenance actions for all components when repairs of components are independent (in problem P4)

\begin{tabular}{|c|c|c|c|c|}
\hline \multirow{2}{*}{ Subsystem $\boldsymbol{i}$} & \multicolumn{4}{|c|}{ Component $\boldsymbol{j}$} \\
\cline { 2 - 5 } & $\mathbf{1}$ & $\mathbf{2}$ & $\mathbf{3}$ & $\mathbf{4}$ \\
\hline $\mathbf{1}$ & $2 \rightarrow 3$ & $0 \rightarrow 1$ & $1 \rightarrow 3$ & - \\
\hline $\mathbf{2}$ & $2 \rightarrow 3$ & $2 \rightarrow 3$ & - & - \\
\hline $\mathbf{3}$ & $0-0$ & $2 \rightarrow 3$ & $2 \rightarrow 3$ & $1 \rightarrow 3$ \\
\hline
\end{tabular}

In comparison with problem $\mathbf{P 1}$ in Example 1, there are two different maintenance activities on component $(1,2)$ and $(3,2)$. Component $(1,2)$ is repaired to state 1 and component $(2,3)$ is repaired to the perfect working state 3; the other components in the system receive similar maintenance actions as in the previous example. The total cost of this strategy is 48 cost units, which indicates that we need more budget for all maintenance actions in comparison with the solution in problem P1. The reliability of the system at level $a=3$ is $R s(3)=0.8757$ which is, interestingly, greater than the optimal reliability in Example 1. The achieved reliability at each level satisfying the reliability constraints is provided in Table 6 . 
Table 6. The probability of the system being in state $k$ or above

when repairs of components are independent (in problem P4)

\begin{tabular}{|c|c|c|c|}
\hline$K$ & 1 & 2 & 3 \\
\hline$R_{S}(k)=P\left(\Phi_{s} \geq k\right)$ & 0.99688 & 0.96446 & 0.8757 \\
\hline
\end{tabular}

Example 3a: Consider the problem in Example 1 again, but there are advantages of repairing multiple components, i.e. the components are economically dependent. The time and cost savings for each component due to the share of setting up of 0.4 time units and 0.8 cost units; the cost saving coefficients of components in subsystem 1, 2, 3 are $0.7,0.6,0.45$; and the time saving coefficients of components in subsystem 1, 2, 3 are $0.5,0.4,0.3$ respectively.

Now, we have to deal with problem $\mathbf{P 1}$ to find the most reliable system with the available time and cost of 25 and 45 respectively. The GA program gives the state vector of components at the time of exiting the maintenance depot as follows:

$X=\left[\begin{array}{lllllllll}3 & 3 & 3 & 3 & 3 & 1 & 3 & 3 & 3\end{array}\right]$

Table 7. The optimal maintenance actions for all components when repairs of components are dependent

\begin{tabular}{|c|c|c|c|c|}
\hline \multirow{2}{*}{ Subsystem $\boldsymbol{i}$} & \multicolumn{4}{|c|}{ Component $\boldsymbol{j}$} \\
\cline { 2 - 5 } & $\mathbf{1}$ & $\mathbf{2}$ & $\mathbf{3}$ & $\mathbf{4}$ \\
\hline $\mathbf{1}$ & $2 \rightarrow 3$ & $0 \rightarrow 3$ & $1 \rightarrow 3$ & - \\
\hline $\mathbf{2}$ & $2 \rightarrow 3$ & $2 \rightarrow 3$ & - & - \\
\hline $\mathbf{3}$ & $0 \rightarrow 1$ & $2 \rightarrow 3$ & $2 \rightarrow 3$ & $1 \rightarrow 3$ \\
\hline
\end{tabular}

In this strategy, we repair the first component in subsystem 3 to state 1 and all other components to the best condition. In comparison with problem P1 in example 1, there are two more maintenance actions which can be taken on components $(3,1)$ and $(3,2)$ within 
available resources.

Table 8. The probability of the system being in state $k$ or above when repairs of components are dependent

\begin{tabular}{|c|c|c|c|}
\hline$k$ & 1 & 2 & 3 \\
\hline$R_{s}(k)=P\left(\Phi_{s} \geq k\right)$ & 0.99734 & 0.98333 & 0.91949 \\
\hline
\end{tabular}

Due to the time and cost savings of repairing multiple components, the achieved reliability of the system at each level (see Table 8) is much higher than the results in Example 1.

Example 3b: In this example, we use three different saving models in section 2.3.2 for modeling the economic dependence when repairing multiple identical components in a sub-system. The input data from Example $3 \mathrm{a}$ is used.

We use the Matlab program to solve the selective maintenance problem to get the most reliable system in the next mission within available time of $T_{0}=25$ units and budget of $C_{0}=45$ units. In this example, the exponents $\beta_{C}^{i}, \beta_{T}^{i}$ for the cost and time savings are set based on $f_{C}^{i}, f_{T}^{i}, \Delta c_{s}, \Delta t_{s}$ so that the adjusted repair cost/time of a component from the proposed model is equivalent to the amount of cost/time for repairing an additional identical component in a sub-system from equation (14). The obtained results of vector $X$ from GA, the total time and cost used for maintenance and the system reliabilities with corresponding parameters used in each model are shown in Table 9.

Table 9.Results of different saving models

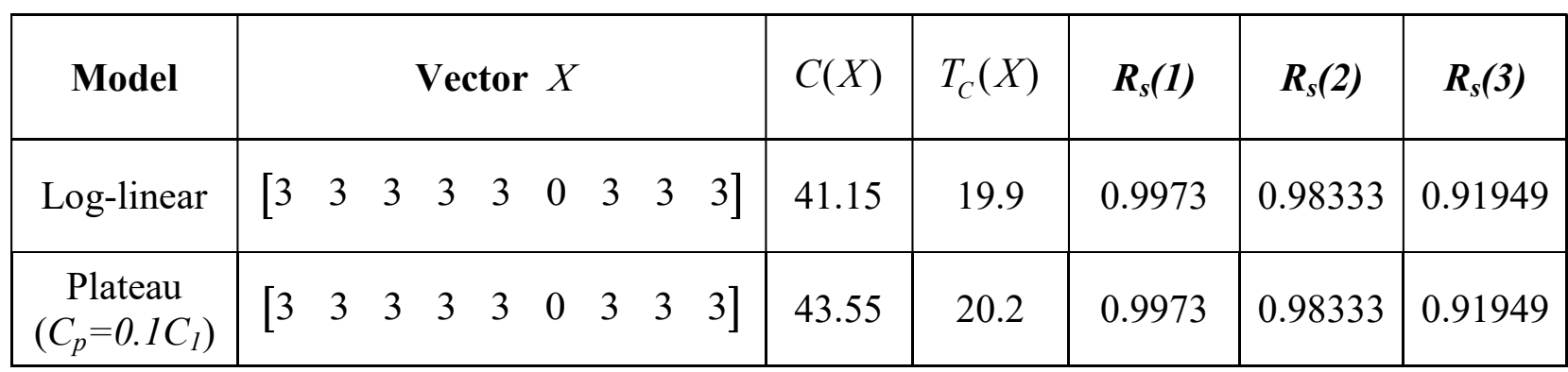




\begin{tabular}{|c|c|c|c|c|c|c|c|c|c|c|c|c|}
\hline $\begin{array}{l}\text { Exponential } \\
\qquad(\mathrm{c}=0.1)\end{array}$ & {$\left[\begin{array}{ll}3 & 3\end{array}\right.$} & 3 & 3 & 3 & 0 & 3 & $\left.\begin{array}{ll}3 & 2\end{array}\right]$ & 41.21 & 18.69 & 0.99725 & 0.98222 & 0.85995 \\
\hline $\begin{array}{c}\text { Proposed } \\
\text { model }\end{array}$ & {$\left[\begin{array}{ll}3 & 3\end{array}\right.$} & 3 & 3 & 3 & $\underline{1}$ & 3 & $\left.\begin{array}{ll}3 & \underline{3}\end{array}\right]$ & 43.75 & 20.7 & 0.99734 & 0.98333 & 0.91949 \\
\hline
\end{tabular}

The first five elements in state vectors, $X(1)$ to $X(5)$, at the time of exiting the maintenance depot are similar in all four models, i.e. the maintenance actions for these components are exactly the same. In the maintenance strategy from the proposed model, one additional maintenance action (corresponding to $X(6))$ can be performed in comparison with Log-linear and Plateau models and two more maintenance actions (corresponding to $X(6)$ and $X(9))$ can be performed in comparison with the Exponential model. The total maintenance cost and time spending in the proposed model is higher than in the other models (43.75 cost unit and 20.7 time units), and this brings the system to a state with higher system reliabilities of $0.99734,0.98333,0.91949$ at levels 1,2 , and 3 respectively.

To explain the differences in the results in Table 9, it is observed that the cost saving of a component in sub-system $i$ is characterized by two parameters, $f_{C}^{i}$ and $\Delta c_{s}$, in the proposed model. Meanwhile, the other saving models describe the economic dependence relationship using a power function and parameter $\beta$. These models can only describe the saving mechanism within a sub-system (when the components are identical), while the proposed model also addresses the economical dependence when repairing components in different sub-systems. This is close to the practical situation since the time and cost of doing common preparation and maintenance activities on the serial-parallel system such as erecting, cleaning, doing inspections, lubricating, etc. can be saved even with different types of components.

To investigate the time and cost savings versus the number of components involved in a maintenance strategy, we study the selective maintenance strategy resulting from Example 3a for independent repair and dependent repair cases. Figure 6 shows how the maintenance 
time and cost for the series-parallel system are saved.

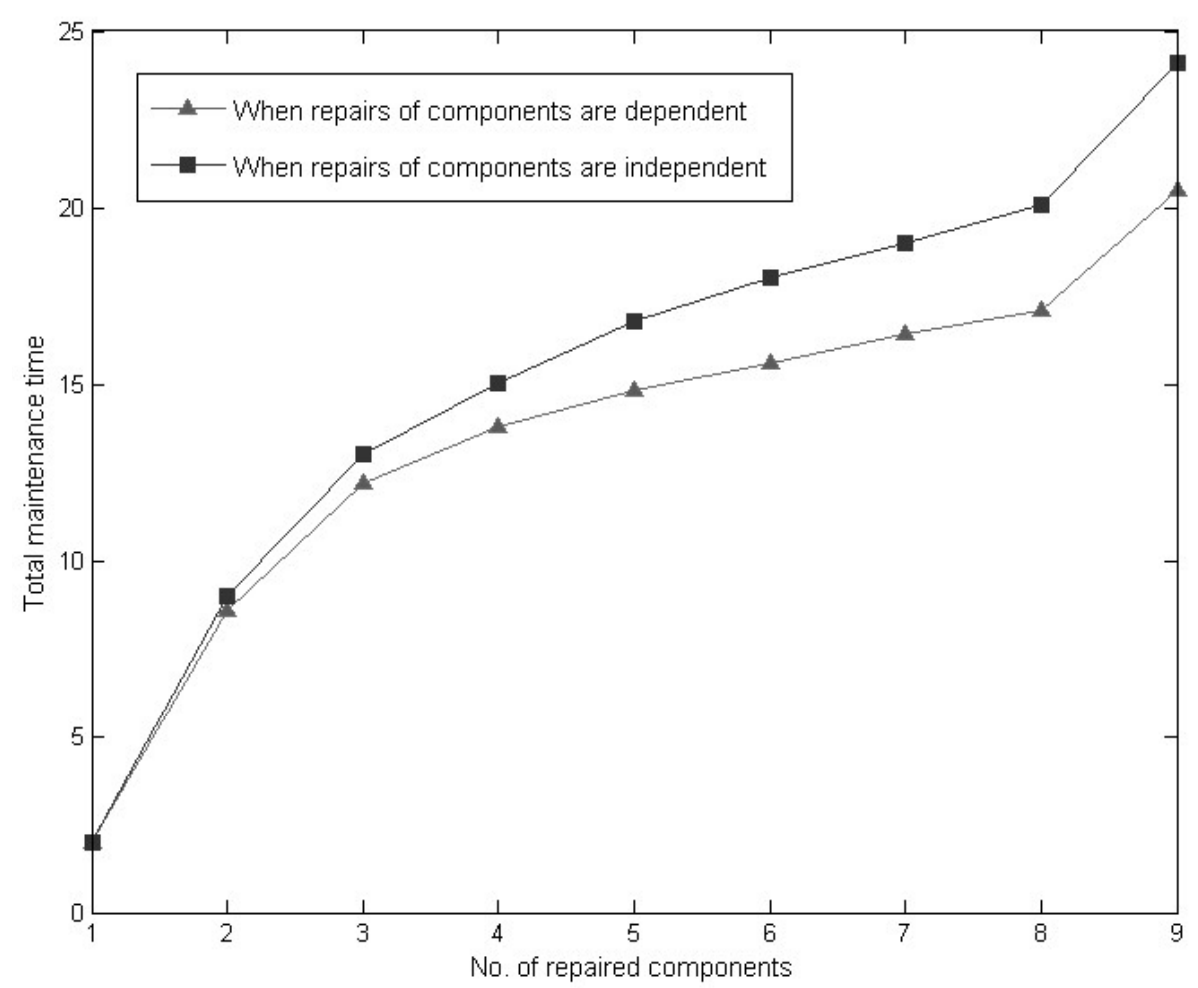

(a)

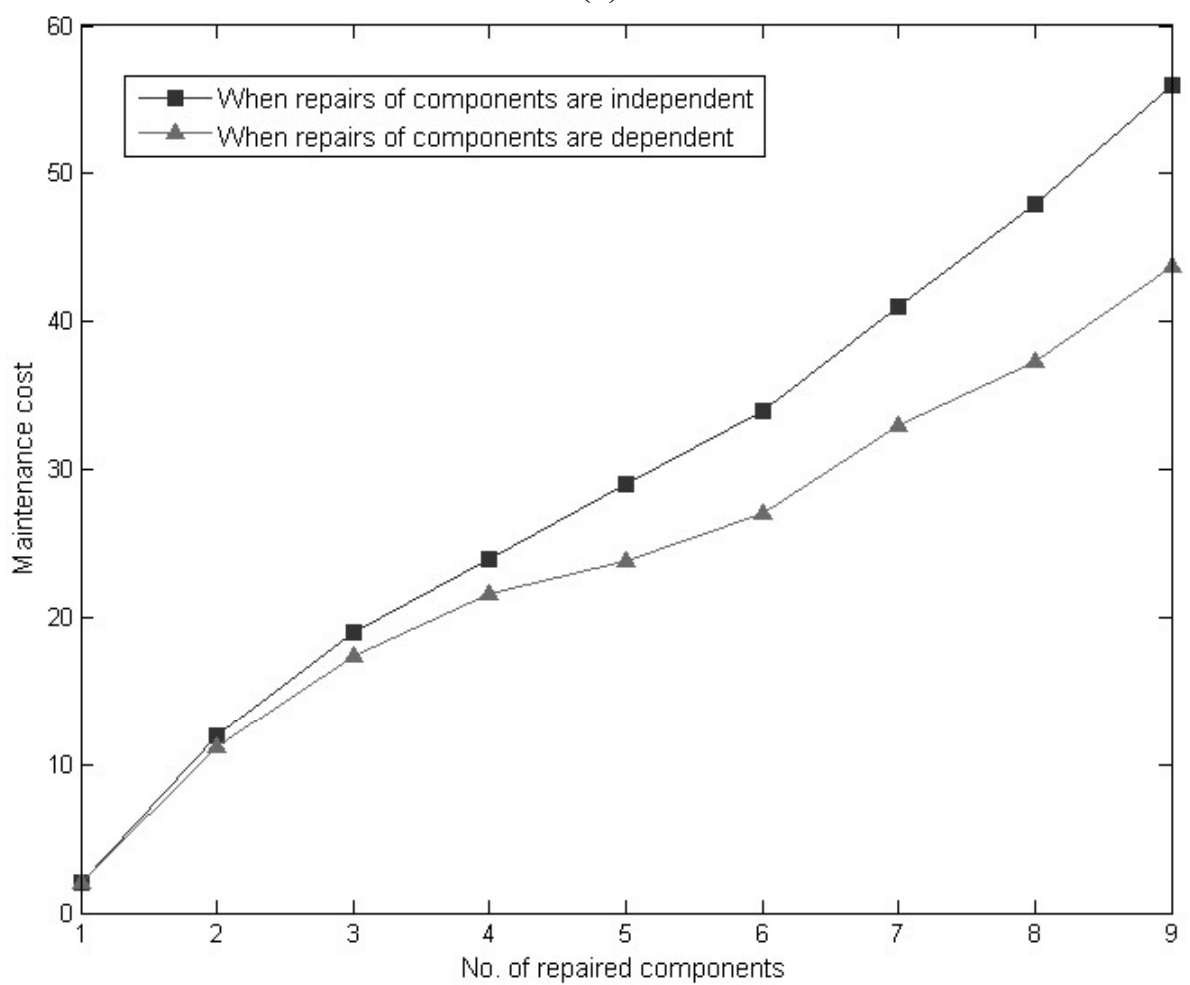

(b)

Figure 6. Total maintenance time (a) and cost (b) of repairing dependent components vs. independent components

Generally, we only need an assumption that the components in the same sub-system have to 
be maintained in a group to gain the benefits of time and cost savings. In Figure 6, we also assume that the components are maintained in an order from the first sub-system to the last sub-system. In this example, there are 9 components which are subjected to be maintained in the maintenance scenario. When the repairs of components are dependent, the maintenance time and cost are smaller than those in the case of independent repairs. The different amounts of required resources between the two cases are more considerable as the number of repaired components increases. To repair all components in this maintenance strategy, the total required time and cost are just 20.7 time units and 43.75 cost units when repairs of components are dependent in comparison with 24 time units and 56 cost units in the case of independent repairs between components.

\section{Conclusion}

This paper proposes the selective maintenance model for multi-state series-parallel system with economic dependence. In the optimization models, the total system maintenance cost, completion time or system reliability can be either treated as objectives or constraints in the mathematical model; different alternative optimization models are derived. The models are closer to practical applications when the maintenance resources for repairing each component are analyzed with regard to the economic dependence. Both time and cost savings can be assured when several components are maintained in a selective maintenance strategy. The economic dependence is analyzed in multi-state contexts based on two types of time and cost savings: (i.) the share of setting up and (ii.) the advantage of repairing multiple identical components in series-parallel systems.

Genetic Algorithms is used to solve the optimization models. The illustrative examples show that the maintenance schedulers may perform different maintenance actions on the system depending on the main objective and the availability of resources. 
In selective maintenance of multi-component systems, the amount of resource savings is system and component specific, i.e. it depends on the nature of the system, number of components and type of components involved. By dividing the type of time and cost savings into fixed (the share of the setting up) and variable (identical repairs), the proposed model can capture both the system features and the component specific. Our main objective is to investigate the selective maintenance problem for multi-state systems under the effect of this saving mechanism. In general, the selective maintenance model in this paper can help the maintenance manager determine the best maintenance strategy to get a reliable system and allocate the resources effectively.

This paper bases on the multi-state series-parallel systems with identical and s-independent components in the subsystem; the system performs missions in periodic intervals. The selective maintenance optimization for multi-state systems with non-identical and/or stochastic dependent components as well as systems with non-periodic operating intervals are recommended for future research.

\section{Acknowledgement}

This research was supported by Natural Sciences and Engineering Research Council of Canada (NSERC) and Vietnam International Education Development (VIED). Comments and suggestions from reviewers and the Editor are very much appreciated.

\section{References}

[1]. Rice W.F., Cassady C.R. and Nachlas J.A., Optimal Maintenance Plans under Limited Maintenance Time, Industrial Engineering Research Conference, 1998.

[2]. Cassady C.R., Pohl E.A. and Murdock W.P., Selective Maintenance Modeling for Industrial Systems, Journal of Quality in Maintenance Engineering, 2001, Vol. 7, No. 2, p. 104-117 
[3]. Schneider K. and Cassady C.R., Fleet Performance under Selective Maintenance, RAMS,2004

[4]. Pandey M., Zuo M.J., Moghaddas R., and Tiwari, M.K., Selective Maintenance for Binary Systems under Imperfect Repair, Reliability Engineering and System Safety, 2013, vol. 113 , p. $42-51$.

[5]. Maillart L.M, Cassady C.R., Rainwater C. and Schneider K., Selective Maintenance Decision-Making over Extended Planning Horizons, IEEE Transactions on Reliability,2009, Vol.58, No.3, p.462-469.

[6]. Chen C., MengM. Q-H. and Zuo M.J., Selective Maintenance Optimization for Multi-State Systems, Proceedings of the IEEE Canadian Conference on Electrical and Computer Engineering, 1999

[7]. Liu Y. and Huang H.Z., Optimal Selective Maintenance Strategy for Multi-state Systems Under Imperfect Maintenance, IEEE Transactions on Reliability, 2010, Vol.59, No.2, p.356-367.

[8]. Archibald TW., Dekker R.,Modified Block-replacement for Multiple-component Systems, IEEE Transactions on Reliability, 1996,Vol.45, p.75-83

[9]. Assaf D. and Shanthikumar J.G.,Optimal Group Maintenance Policies with Continuous and Periodicinspections,Management Science,1987,Vol.33, p.1440-1452

[10]. Wildeman R.E., Dekker R. and Smit A.C.J.M., A Dynamic Policy for Grouping Maintenance Activities, European Journal of Operation Research, 1997, Vol. 99, No. 3, p.530-551.

[11]. Okumoto K. and Elsayed EA., An Optimum Group Maintenance Policy, Naval research Logistics Quarterly, 1983, Vol. 30, p.667-674

[12].Dekker R., Roelvink IFK.,Marginal Cost Criteria for Preventive Replacement of a Group ofComponents, European Journal of Operational Research,1995, 84:467-480

[13]. Dekker R., Wildeman R., and Van Der Duyn Schouten F.A., A Review of Multi-Component Maintenance Modelswith Economic Dependence, Mathematical Methods of Operations Research, 1997,Vol. 45, p.411-435

[14]. Nourelfath M. and Chatelet E., Integrating production, inventory and maintenance planning for a parallel system with dependent components, Reliability Engineering and System Safety, 2012, Vol.101, p.59-66.

[15]. Maaroufi G., Chelbi A., and Rezg N., Optimal selective renewal for systems subjected to propagated failures with global effect and failure isolation phenomena, Reliability Engineering and System Safety, 2013, Vol.114, p.61-70.

[16]. Xue J. and Yang K., Dynamic Reliability Analysis of Coherent Multistate Systems, IEEE Transaction on Reliability, 1995, Vol.44, No.4, p.683-688. 
[17]. Anzanello J. M. and Fogliatto S.F., Selecting the best clustering variables for grouping mass-customized products involving workers' learning, International Journal of Production Economics130, 2011, pp 268- 276

[18]. Jaber Y.M., Learning Curves Theory, Models, and Applications, CRC Press, 2011

[19].Goldberg, David E., Genetic Algorithms in Search, Optimization and Machine Learning, Addison-Wesley, 1989

[20]. Affenzeller M., Winkler S. , Wagner S., and Beham A., Genetic Algorithms and Genetic Programming: Modern Concepts and Practical Applications, Chapman \& Hall CRC, 2009 